\title{
Parameter Estimation of Weak LFM in Heavy noise based on Stochastic Resonance and RAT-FrFt
}

\author{
HE Xudong ${ }^{1,2, a}$, LIU Jue ${ }^{1,2}$, Dong Yangze ${ }^{1,2}$ \\ ${ }^{1}$ Science and Technology on Underwater Acoustic Antagonizing Laboratory, Shanghai,201108, \\ China, \\ ${ }^{2}$ Shanghai Marine Electronic Equipment Research Institute, Shanghai, 201108, China \\ ahexudong1983@126.com
}

Keywords: Stochastic Resonance; RAT; FrFt; LFM; Estimation.

\begin{abstract}
Signal parameter estimation is a part of the signal processing, this paper focus on the weak LFM parameter estimation by stochastic resonance, the twice sampling and different step make sure the bistabe system reach to resonance, then the SNR is improved, the high resolution estimation is come from the accumulation to the line by RAT-FRFT, some simulation have been done for testing the method.
\end{abstract}

\section{Introduction}

Signal Parameter is an elementary factory for Signal which is the purpose of detective and identify the target in signal processing. The signal parameter can be attain by STFT, wavelet feature and many other method at high SNR[1], but in lower SNR, the signal is immerge in the noise, above method is invalidation. Extracting low intensity signal from noise is a hotspot in underwater acoustic signal processing[2], some methods have been tested, but it is useful under some condition. This paper bring the Stochastic Resonance to improve the SNR because the signal and noise effect each other in the bistable system[3], the energy of noise would transform into the signal when the signal, noise and system is matching, the SNR would be elevation. It is useful for identification signal. RAT-FRFT assembles the energy of LFM in fractional field, it calculated in three steps and more precise, it is useful for identification LFM signal in low SNR[4].

\section{Theory of Stochastic Resonance}

Stochastic Resonance is a prevalent phenomenon in the nature which is induced by noise because of nonlinear, the elements of the stochastic resonance are noise, signal and bistable system[3].

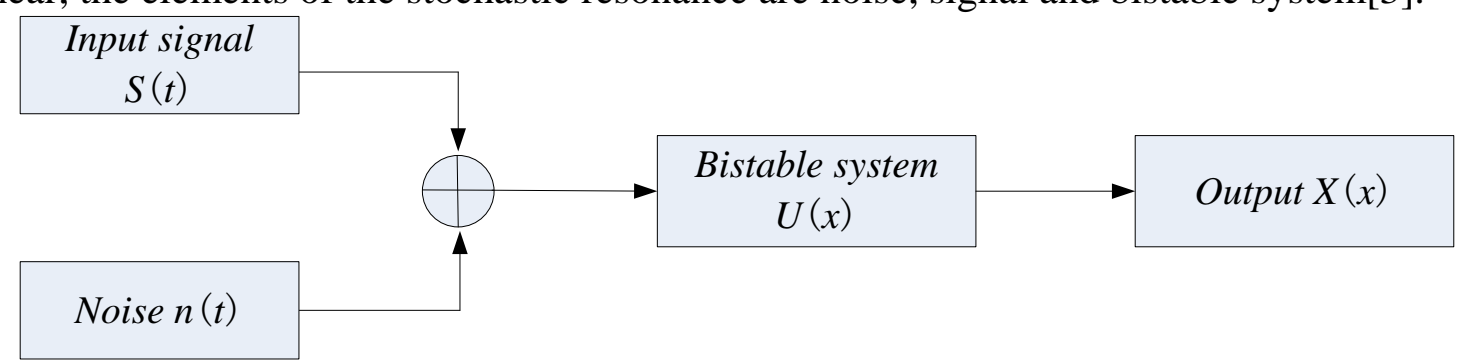

Fig.1 the Stochastic Resonance

The noise indicate the effect on macroscopic variable because the stochastic movement of particle, it present as unorderly movement in macroscopically caused by movement in microcosm. The noise of the system is little for macroscopic system, and then the out noise can be used for affecting to the system which is the present of the out movement. The noise and nonlinear system can be contact by Langevin function [5]:

$$
\dot{x}=f(x)+n(t)
$$

If the Brown particle movement at a dimension in bistable system: 


$$
f(x)=-\frac{d U(x)}{d x}=a x-b x^{3}
$$

Then, the function of bistable system $U(x)$ is a multinomial in 4 order dimensions

$$
U(x)=-\frac{1}{2} a x^{2}+\frac{1}{4} b x^{4}
$$

There are 3 solutions when $f(x)=0: x_{1}=0, x_{2,3}= \pm \sqrt{\frac{a}{b}}$;

Correspondingly, the $U(x)$ have 3 solutions: $U\left(x_{1}\right)=0, U\left(x_{2,3}\right)=\frac{a^{2}}{4 b}$

the 3 solutions is called as rampart highleight.

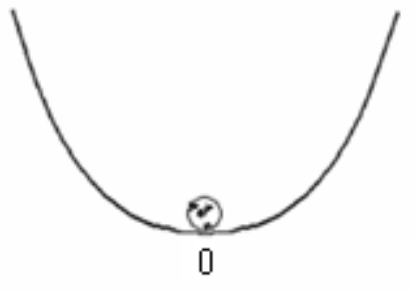

(a)

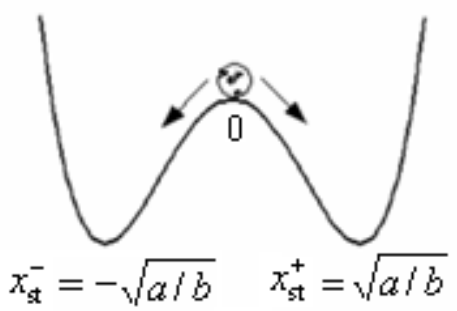

(b)

Fig.2 Sketch map of potential well (a) and double potential well (b)

The particle would transit between two solutions when there is out driver, the transition frequency would be equal to the frequency of out driver $f_{0}$ if the intensity of noise is correctly. Then the stochastic transition between two potential well become orderly transition under the out driver in periodically, which is stochastic Resonance, the energy of noise transform into the signal, the SNR is increased, it is helpful to extracting the weak signal from noise.

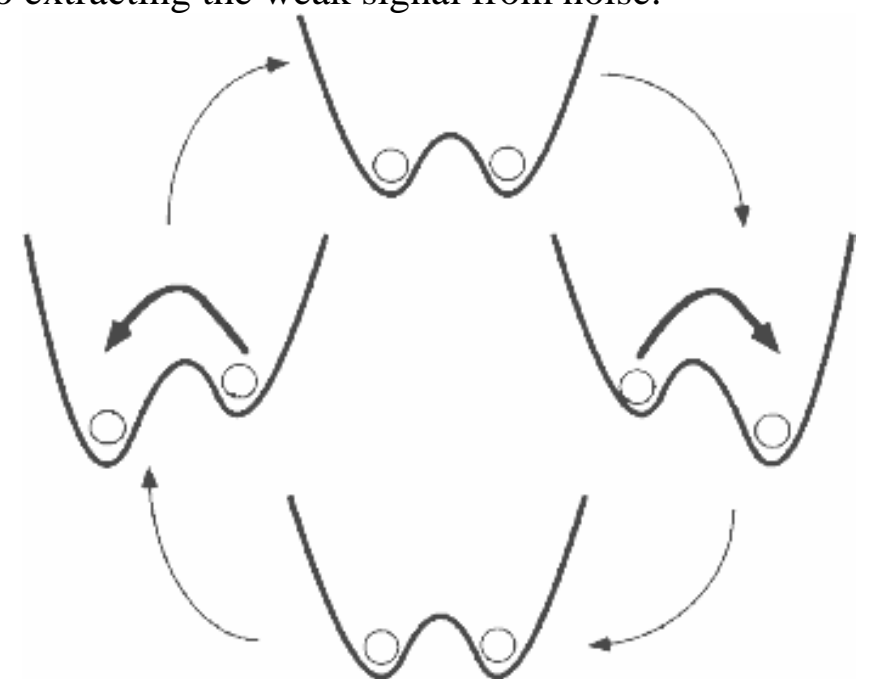

Fig. 3 the bistable potential transit under periodic driving

The four order Runge-Kutta algorithm is used for simulation in this paper, the algorithm is based on Thaler series[6]:

$$
x_{n+1}=x_{n}+\frac{h\left(k_{1}+2 k_{2}+2 k_{3}+k_{4}\right)}{6}
$$

$k_{1} 、 k_{2} 、 k_{3} 、 k_{4}$ is coefficient which can be calculated by formula (5)-(8):

$k_{1}=a x_{n}-b x_{n}^{3}+s_{n}$

$k_{2}=a\left(x_{n}+\frac{k_{1}}{2}\right)-b\left(x_{n}+\frac{k_{1}}{2}\right)^{3}+s_{n}$ 


$$
\begin{aligned}
& k_{3}=a\left(x_{n}+\frac{k_{2}}{2}\right)-b\left(x_{n}+\frac{k_{2}}{2}\right)^{3}+s_{n+1} \\
& k_{4}=a\left(x_{n}+k_{3}\right)-b\left(x_{n}+k_{3}\right)^{3}+s_{n+1}
\end{aligned}
$$

$a 、 b$ is system parameter and the system would be in resonance by $a 、 b$.

\section{Theory of RAT-FrFt}

RAT-FRFT is an identify signal algorithm made up of RAT and FRFT. Radon can extracting the line in picture(formula (9)), it integrate a line parallel to axis $v$ correspond to every $u$ in a new coordinate $(u, v)$ which is obtain by rotating the old coordinate $(x, y)$, then it can be used for extracting the LFM in signal processing because the ambiguity of LFM is Line(formula (10)), while ambiguity indicate the character of the signal in time-frequency domain (formula (11)), the frequency modulate rate also can be calculated by RAT, So, the center frequency can be get by FRFT easily by frequency modulate rate(formula(12)-(15)).

$$
\begin{aligned}
R_{\alpha}(u) & =\int_{-\infty}^{\infty} f(\rho \cos \alpha-v \sin \alpha, \rho \sin \alpha+v \cos \alpha) d v \\
& =\int_{-\infty-\infty}^{\infty} \int_{-\infty}^{\infty} f(\rho \cos \alpha-v \sin \alpha, \rho \sin \alpha+v \cos \alpha) \delta(\rho-u) d \rho d v
\end{aligned}
$$

where $-\infty<u<+\infty,-\frac{\pi}{2}<u<+\frac{\pi}{2}, u$ is horizontal axis and also shows the displacement to the origin, formula (9) express that the integral of function $f(x, y)$ along with a line which distant from the origin $u$, the slope is $\tan \alpha$.

$$
\begin{aligned}
A(\tau, v) & =A^{2} \int_{-\infty}^{+\infty} e^{j 2 \pi(f \tau+k t \tau)} e^{-j v t} d t \\
& =A^{2} \delta(v+2 \pi k \tau) e^{j 2 \pi f \tau} \\
& A(\tau, v)=\int_{-\infty}^{+\infty} x\left(t+\frac{\tau}{2}\right) x^{*}\left(t-\frac{\tau}{2}\right) e^{-j v t} d t
\end{aligned}
$$

FRFT can be seen as a rotate operator in time-frequency plane, the $p$ order Fourier transform of signal shows in formula (12), the angle $\alpha$ is relations with $p$ order as $\alpha=p \frac{\pi}{2}$. FRFT identify the LFM because the frequency modulate rate and center frequency can be calculated by formula (14) and (15), then ,FRFT can distinguish different LFM by different $K$ and $f_{0}$. The center frequency $f_{0}$ can be estimated in one dimension if the $\alpha$ is known.

$$
\begin{aligned}
& X_{p}(u)=F_{x}(p, u)=\int_{-\infty}^{+\infty} K_{p}(t, u) d t \\
& K_{p}(t, u)=\left\{\begin{array}{l}
\sqrt{\frac{(1-j \cot \alpha)}{2 \pi}} e^{j \frac{t^{2}+u^{2}}{2} \cot \alpha-j u t \csc \alpha}, \alpha \neq n \pi \\
\delta(t-u), \alpha=2 n \pi \\
\delta(t+u), \alpha=2 n \pi \pm \pi
\end{array}\right. \\
& \hat{k}_{m}=-\cot \alpha \\
& \hat{f}_{0}=\hat{u}_{0} \csc \alpha
\end{aligned}
$$




\section{Simulation on Stochastic Resonance and RAT-FrFt}

A 0.04 s LFM which cover from $1500 \mathrm{~Hz}$ to $2000 \mathrm{~Hz}$ is used for testing the method, SNR=-20dB. Twice sampling and different step are two method both can adjust the system to resonance, this paper using the two method in same time, the first sample frequency is $f_{s}=100 \mathrm{kHz}$, the step is $h=\frac{1}{160}, a=0.005, b=60$.

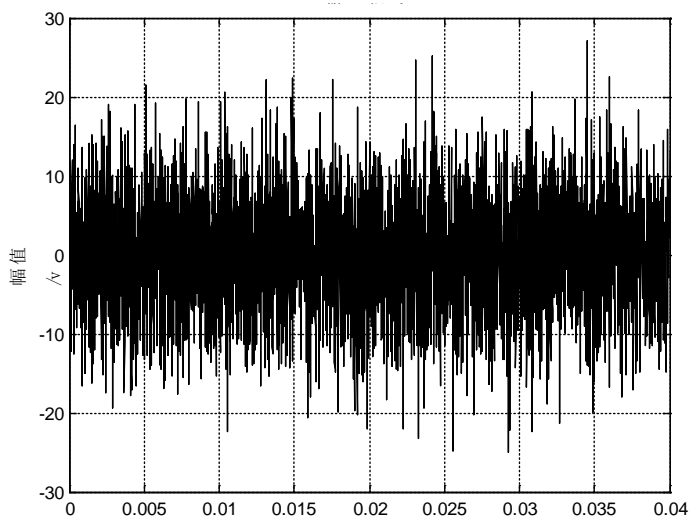

Fig.4 the input signal in time domain

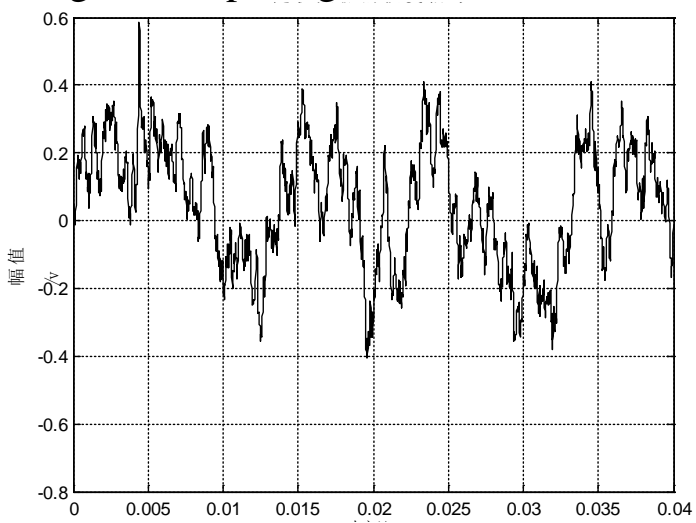

Fig.6 the output signal in time domain

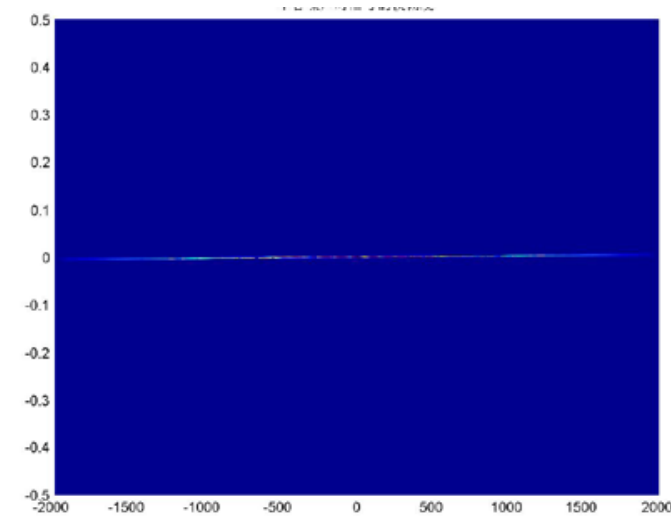

Fig.8 the ambiguity of LFM

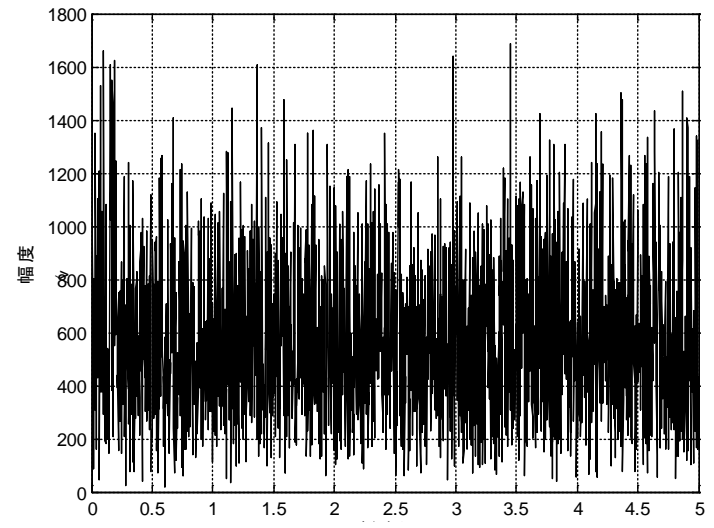

Fig.5 the frequency spectrum of input signal

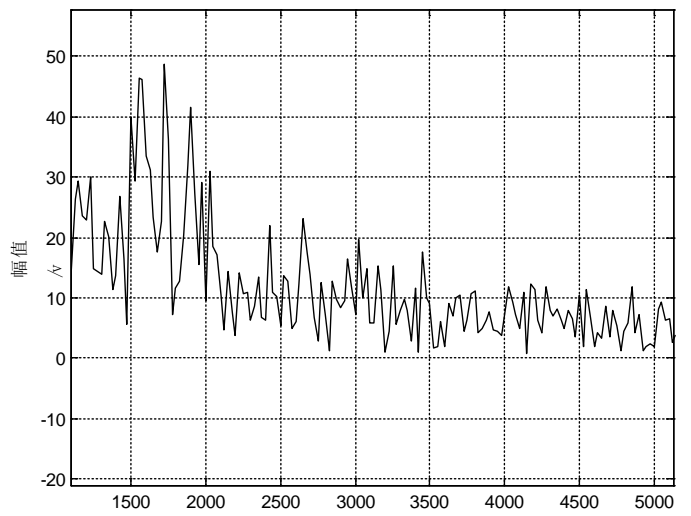

Fig.7 the frequency spectrum of output signal

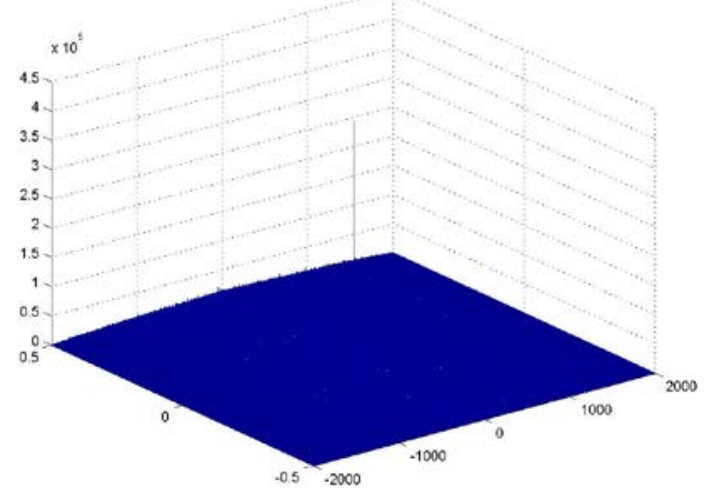

Fig.9 the ambiguity of input signal

The input signal is disorder in time domain and frequency domain, there is nothing character in figure 4 and figure 5 . Figure 6 shows the output signal, the input signal is down sampling at first and Up sampling after the bistable system, the output signal is seem like a sine in whole, it resonate between -2 and 2 in time domain, the spectrum (Figure 7) during $1500 \mathrm{~Hz}$ to $2000 \mathrm{~Hz}$ is higher than others, then the signal in $1500 \mathrm{~Hz}$ to $2000 \mathrm{~Hz}$ can be confirmed, but the parameter of the signal cannot be estimated. Figure 8 shows the ambiguity of LFM, figure 9 shows the ambiguity of input signal, and figure 10 shows the output ambiguity of signal, figure 11 is also shows the output ambiguity of signal in 3D. The ambiguity of LFM is a line in XY plane, but the ambiguity of input signal is a point when noise is heavy, it is not useful for identify the signal, after the bistable system, the output signal shows 
some characters of the original signal, it would help to estimate the parameter although there is some disturb, it may bring error. Figure 12 and figure 13 show the fractional Fourier transform of input and output signal respectively, there is no accumulation in fractional domain because of low SNR in figure 12, it cannot estimate, while there is peak after the bistable system, the signal is accumulation in fractional domain, then, it is useful for estimating.the result of simulation is $k=12551, f_{0}=1755 \mathrm{~Hz}$.

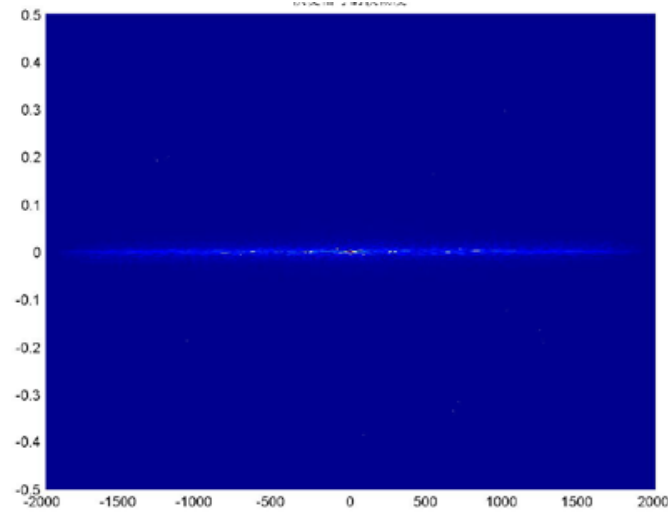

Fig.10 the ambiguity of output signal

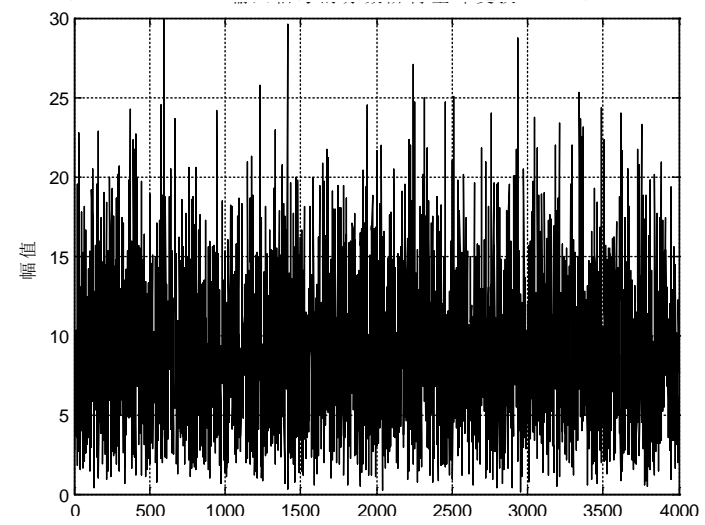

Fig.12 the FrFt of input signal

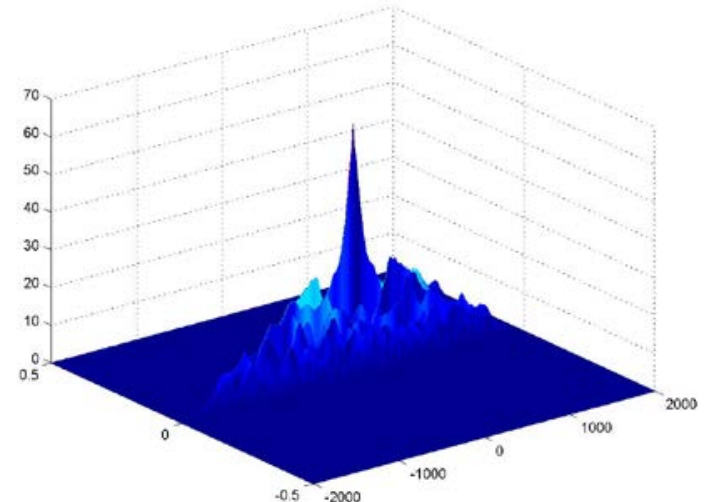

Fig.11 the ambiguity of output signal in 3D

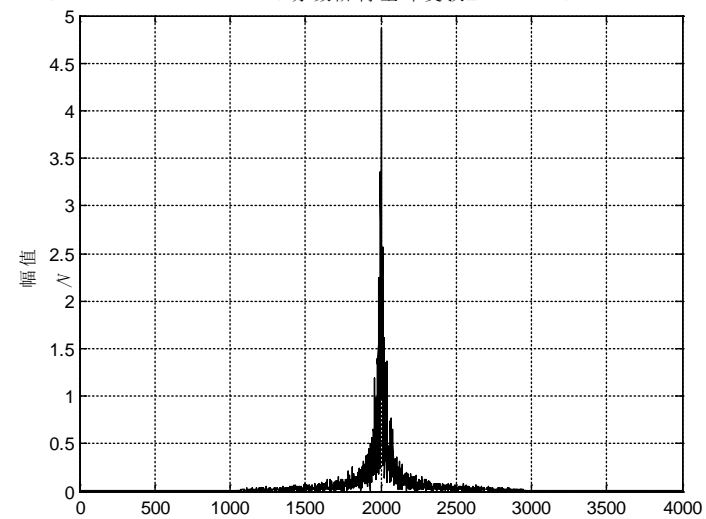

Fig. 13 the FrFTof the output signal

\section{Summary}

This paper focuses on the parameter estimation of wake LFM which submerged in heavy noise, stochastic resonance take the energy of noise transform to signal when the bistable system is resonated, it help to detect the signal, and RAT-FrFt is useful for estimating the parameter in high resolution easily, then the method has been tested with simulation, but it need more research on how to adjust the parameter of bistable parameter to make the system resonance with different SNR and other conditions.

\section{References}

[1] Wang Hongyu: Nonstational signals analyze and processing, 1999,1-15.

[2] Xu Huifa, Liu Feng, Zhang Xin: Detection and parameter Estimation of strong and weak LFM signals in Fractional Fourier Domain,SIGNAL PROCESSING,Vol 27(2011),p.1063-1068.

[3] Zhang Ying:Research on the Mechanism and Methodology of Stochastic Resonance Signal Recovery,TianJin China,2010.

[4] He Xudong,Yin,Mingming,Liu,Jue:Studying on Parameter Estimation of LFM Based on RAT and FrFt,CISP-BMEI 2013.

[5] Benzi R,Alfonso S,Vulpiani A, The mechanism of stochastic resonance,J,Phys.A,1981,14: p.453-457. 
[6] Leng Yonggang,Mechanism Analysis of the Large Signal Scale-transformation Stochastic Resonance and Its Engineering Application Study,2004. 